Pev. Chil. Pediatr. 67 (3); 121-124, 1996

\title{
Experiencia nacional en el manejo del síndrome de intestino corto en niños
}

\author{
Carlos Castillo D.'; Silvia Guardia B. '; Teresa Young A.'; \\ Pedro Herskovic L. '; Angel Fuentes Z.'; Abdallah Harún M. '; Marcelo Ducheylard R. '; \\ Carolina Laguna N. '; Elisa Aguila G. ': Guillermo Correia D. ': Sylvia Ibáñez T.'; \\ Danicl Abumohor D. '; Verónica Marín B. ', M. Jesús Rebolto G. ${ }^{\text {' }}$
}

\section{Short bowel syndrome}

\begin{abstract}
To delineale loca ccuses, maragement and shor term (first $\$ 0$ days after intestinal resectio-) :esulis, a ret. rospective colaborative study on pediatric cases of short bowel syndrome after intestinal surjery seen from year 1989 throughout 1995 at six sole cnd one privale chilean hospitals was done. Fourleen fatients with small bovei remnanits shorer than $50 \%$ of normelly expected for gesiational ar chro-oiogical oge were avalable tor review. Treir initial

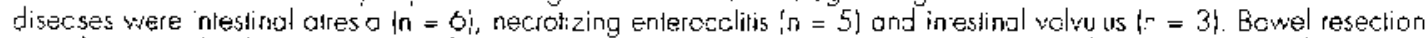
was done manly along the neo-atci period Most palients had been prematurely born infarts $(10 / 14)$ and 10 wee male. All subeets showed long hospitel courses lal leost 3 montrsl. and parenteral nutrition was given to all hem

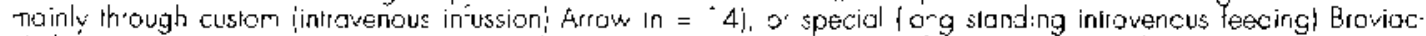

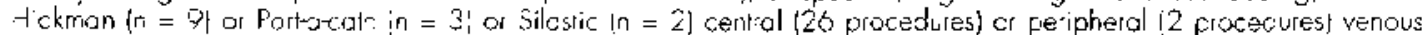

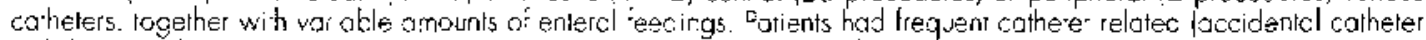
withdrawal. obstruction, thromb, hemolome, sepsis); me abolic 'hypoglicemia, ryferglicerria, hyperrigivceridemici or

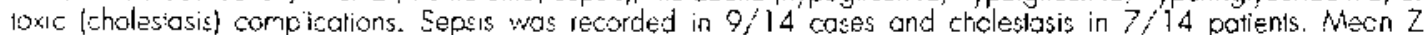
scores of weight/age and length/age remainec under -2 nirely doys afier inlestirial resenticn in soite of parenteral leeding. Meon caily money expense of parenteral leeding was approximciely US 25 dollars. Highly soecialyzed learns and aporopriple caheters and guidelines cre coally at need to rake care of these patients.
\end{abstract}

(Key words: shot bowal syndrome, i-traverous eecoing.!

El síndtome de intestino corto se ha definido como la longitud funcional del intestino delgado distal al ángulo de Treitz, menor a $50 \%$ de su largo total normal'. La mayoría de los casos comunicados se originan en el período nconatal debido a malformaciones congénitas o enfermedades intestinales adquiridas a esa edad -principalmente enterocolitis necrótica- que causan resecciones quirúrgicas masivas ${ }^{2}$.

El mejoramiento de las técnicas quirúrgicas, de los cuidados intensivos y particularmente de

I. Grupo colaborativo pediárico en manejo de intestimo corto:

Hospitales Sótero del Río. Luis Calvo Mackenna, San Juan de Dios. San Borja-Arriacán. Roberto del Río, Clinica Santa Maŕa, de Santiago, y Carlos Van Buren, de Valparaíso, e Instituto de Nutrición y Tecoolugía de los Alimentos (INTA de la Universidad de Chile. los métodos especiales de asistencia nutricional. han permitido que estos pacientes sobrevivan más allà del período agudo de la enfermedad ${ }^{3}$. Se ha logrado, incluso, la adaptación del intestino remanente para cumplir todas las funciones propias sin tener que recurrir a alimentación parenteral complementaria 4 .

Dado que este sindrome es poco frecuente en los hospitales chilenos, es difficil alcanzar por separado una experiencia satisfactoria con su mancjo, lo que ha motivado la constitución de un grupo de trabajo que permita conocer la realidad local y obtener la información necesaria para mejorar las prácticas clinicas sobre esta materia en el país. El objetivo de este estudio fue describir las principales causas y analizar el manejo del síndrome de intestino corto en las fases iniciales de su evolución en un conjunto de centros relevantes del país. 


\section{Pacientes y Método}

Se cstudiaron recrospecivamente los antecedentes de todos los pacientes pedíáticos sometidos a resecciones de más de $50 \%$ de la longitud estimada normal para el intestino delgato. para la edad gestacional y cronológica, registrados enste los años 1989 y 1995 en los hospitales Luis Calvo Mackenna. Sótero del Río. San Juan de Dios, San Borja-Atriarán, Robcito del Rí y Clinica Sauta María de Santiago, y Carlos Van Buren, de Valparaíso, consignando la edad gesracional, el motivo de las resecciones. asi como también la evolución clínica y antroponétrica en los priuneros tres meses despues de la operacion, incluyendo las complicaciones intecciosas. de los caléteres intravenosos y la via de instalación: trastomos metabólicos: frecuencia de colestasia y leralidad. El peso y la talka se analizaron como puntajes $Z$ de las tablas de la Organización Mundial de Salud (OMS) y el Centro Estadounidense de Estadisticas dc Salud (NCHS) al momento de la resección intestinal y 90 dias después. Se estudió ademas les costes de preparación y administración de las alimentaciones parenterales. tomando el perfil del valor de los produccos vendidos a los hospitales públicos expresado en pesos. moneda chitena de 1995.

\section{Resultados}

Catorce niños de los siete hospitales cunsplían los criterios exigidos de intestino corto. Su cdad -en el momento del análisis- era de 3 meses a 10 anos, promedio seis meses. Diez eran varones, diez habían nacido antes del término de la gestación y doce habían sufrido la complicación que causó la intervençión quirúrgica en los períodos de recién nacido o lactante menor (antes de la edad de un año).

Las causas del síndrome de intestino corto fueron principalmente atresia intestinal, que se registró en seis casos, en uno de ellos asociada con gastrosquisis y en otros dos con la modalidad en cáscara de marizana (aple peel); la en. terocolitis necrótica lo fue en cinco casos y el volvulo intestinal en tres. El remanentc de intestino delgado distal al ángulo de Treitz era menor que $50 \mathrm{~cm}$ en 9 casos y mayor en los 5 restantes (márgenes 4 y $90 \mathrm{~cm}$ ); en 7 pacientes la resección incluía la válvula ileocecal y en 4 se habia hecho resección parcial del colon.

Después de un curso postoperatorio dificultoso en todos los enfermos, unos quedaron bajo la responsabilidad de equipos de manejo nutricional especializado, en determinados hospitales, y otros a cargo de profesionales individuales (médico, onfermera, nutricionista). A todos se les instaló, para alimentación parenteral. catéteres intravenosos que debieron ser cambiados con frecuencia. En la mayoría de los 28 procedimientos (26 en vía venosa central y 2 en venas periféricas), se emplearon catéteres corrientes (Arrow, $n=14$ ) al no disponerse de otros más adecuados, que se instalaron en venas centrales, como cava superior o inferior; en los restantes se usaron catéteres especiales de larga duración de Hickman-Broviac (n = 9), Porth-acath $(n=3)$ o de Silastic $(\mathbf{n}=2)$. Todos Ios niños recibieron alimentación parenteral desde los primeros días del periodo postoperatorio, agregándose, dentro de los siguientes 30 días, alimentación enteral, de modo que al cabo de 90 días todos cstaban recibiendo alimentación parenteral (principalmente) y cnteral (en volúmenes no siempre bien registrados en la ficha (ínica).

Los pacientes sufrieron numerosas complicaciones en el transcurso de 10 90 días siguientes a la operación. Estas fueron principalmente de tipo técnico (obstrucciones, retiro accidental de catéteres), metabólico (hiperglicemias, hipoglicemias, hipertrigliceridemias) y otras cuyo registro no fue los suficientemente estricto como para su cuantificación precisa. No obstante lo anterior, las complicaciones de mayor significado y gravedad fueron colestasia, observada en siete casos, y sepsis, que afectó a nueve pacientes: con un episodio en tres de ellos, dos veces en cuatro casos y en tres oportunidades en los dos restances, lo que se comprobo clínicamente y por ef laboratorio. Las bacterias identificadas eran principalmente Staphylococcus epidermidis y Staphylococcus aureus, como también Klebsiella pnemoniae y Escherichia coli. Puesto que en la mayoría de los hospitales encuestados no sc habían desarrolado normas al respecto, no fue posible determinar si tales aislamientos obedecían a los requisitos de la detinición de sepsis por catéter.

La cvolución nutricional no mostró tendencia a la mejoría en el curso de los noventa días siguientes a la intervención, a partir de un promedio inicial de puntaje $Z$ inferior a -2 , en las relaciones de pesotedad y talla/edad.

Los costes de la alimentación parenteral en el hospital, para un lactante de 6 meses (edad promedio de los enfermos estudiados) correspondían a \$ 5750 diarios (en moneda chilena de 1995) en soluciones y bolsas de alimentación parenteral. más $\$ 4512$ diarios en catéteres intra- 
venosos, bajadas de vena, mantención de bombas de infusión y de la campana de flujo laninar (utilizada en la mayoría de los hospitales para la preparación aséptica de las soluciones). Esto suma \$297860 mensuales por paciente, sin considerar el valor de un día cama y los gastos en personal, alimentación cnteral, exámenes de laboratorio, desarrollo e incorpotación de nueva tecnologia, control de calidad de las preparaciones, mancjo de las complicaciones intercurrentes.

No se registraron muertes en el limitado período de 90 días incluido en este análisis. pero posteriormente cuatro de los pacientes han fallecido como consecuencia de infecciones o colestasia. Dados la variable cvolución de eslos anfermos, que pueden cursar en varios años y otros antecedentes ya disponibles en algunos de los casos, la letalidad probablemente será mayor.

\section{Comentario}

Esta casuística muestra el importante trabajo hospitalario que exige una enfermedad crónica como esta, de baja frecuencia, pero de alto impacio emocional para la familia y elevada inversión social y cconomica en recursos profesionales. de estructura y organización.

Aunque no conocemos la prevalencia del síndrome de intestino corto, su frecuencia para el total dc los centros hospitalarios participantes fue de dos casos por año. La evaluación realizada no permite tampoco hacer afirmaciones acerca de Ios eventuales efectos del manejo médien y quirúrgico neonatal y postneonatal en ta disminución o aumento del número de casos de intestino corto.

El buen éxito en el manejo de este tipo de enfermos se expresa, a largo plazo, en la maxima adaptación del intestino remanente para digerir y absorber todos bos nutrientes requeridos por el niño. si es posible habiendo restado to más precozmente posible el apoyo de alimentaciốn parenteral ${ }^{3.4}$. Un resultado favorable incluye también cierta adaptación del colon, para efectuar alguna absorción de los ácidos grasos de cadena corta producidos por la flora bacteriana a partir de hidratos de carbono ${ }^{56}$, pero. además, con mínimo de complicaciones metabólicas, técnicas e infecciosas durante la cvolución.
La adaptación total o parcial del intestino se puede conseguir en un plazo máximo de alrededor de dos años, especialmente en los niños con síndrome de intestino ultracorto (intestino remanente de menos de 60 o $70 \mathrm{~cm}$ ). Posteriormente es poco probable que ella mejore $y$, en esta situación, es posible aplicar técnicas quirúrgicas tendientes a alargar el intestino remanente, si bien el trasplante todavía es una alternativa poco segura y fucra de alcance en el país? La buena sobrevida en los 90 días analizados no es un buen retlejo de los resultados a largo plazo, como lo ilustra la muerte posterior de algunos de ellos por complicaciones infecciosas o colestasia.

Entre los factores que claramente exigen mejorar el mancjo cstán las complicaciones infecciosas, en particular las sepsis por catéter. El elevado riesgo de estas infecciones, en los primeros 90 días del período postoperatorio, tiene entre sus causas centrales el uso inadecuado de catéteres venosos centrales ${ }^{8}{ }^{9}$, principalmente por fallas en la selección de éstos, en parte por problenuas de costo, pero también por ausencia de normas locales adecuadas, y puede subsanarse con el empleo de catéteres para uso prolongado en vías venosas centrales (HickmanBroviac y Porth-a-cat), como ya es habitual en atgunos centros, y la aplicación de protocolos de mancjo ${ }^{10}$. En otros países el riesgo de infecciones ha llevado a extremar las medidas preventivas, que incluyen manejo por equipos especializados: técnicas asépticas; uso de equipos estériles para cambiar las soluciones de alimentación parenteral y empleo de campana de flujo laminar en su preparación. Uno de los mayores avances ha sido el establecemiento de un programa de asistencia nutricional en el hogar ${ }^{3}, 11.12$. Con ello se han logrado frecuencias de sepsis por catéter del orden de una por niño por año $0^{40}$, junto a evidente mejoría en el desarollo psicomotor del enfermo y en las relaciones familiares.

La colestasia es otra complicación de importancia en los casos presentados. Los factores considerados contribuyentes a ella estaban presentes en los pacientes de esta seric en que ocutriô: prematurez, alta frecuencia de infecciones, aportes a veces poco apropiados de aminoácidos y glucosa ${ }^{13-15}$. Es posible controlar una buena parte de estos fuctores con protocolos más rigurosos de manejo, incluyendo la alimentación 
parenteral en ciclos, es decir, administrada por períodos limitados de tiempo cada día, dejando el catéter tapado y heparinizado por algunas horas. Puesto que aún así siguen produciéndose nuevos casos de colestasia, se están intentando además tratamientos curativos o paliativos de ella, con resultados aún no concluyentes, entre ellos el ácido ursodeoxicólico y la colecistoquinina ${ }^{\text {4. } 15}$.

El intercambio de experiencias entre centros hospitalarios del país y con los de otras naciones debería contribuir a mejorar el pronóstico vital y a disminuir las complicaciones en los niños con síndrome de intestino corto. Un protocolo prospectivo de manejo ciertamente facilitaría el conocimiento de su evolución y los hechos que inciden en ella.

\section{Resumen}

En un estudio colabotativo retrospectivo se analizó la evolución hasta 90 dias después de la resección intestinal de 14 niños con intestino corto registrados en siete hospitales (seis estatales) chilenos entre los años 1989 y 1995. La causa de la resección fue en seis casos atresia intestinal, en cinco enterocolitis necrosante y en tres vólvulo y se realizó, en casi todos, en el periodo de recién nacido. Los pacientes cran preferentemente prematuros (10/14) al nacer y de sexo masculino (10/|4). Todos requirieron hospitalización prolongada (mínimo 3 meses), alimentación purenteral, principalmente por via venosa central, a través de catéteres venosos corientes Arrow $(n=14)$, o especiales BroviacHickman $(n=9)$, Port-a-cath $(n=3)$ y Silastic $(\mathrm{n}=2)$, con volúmenes variables de alimentación enteral. Las complicaciones fueron trecuentes, debidas a causas técnicas (obstrucción o salida del catéter, trombos, hematomas, sepsis); metabólicas (hipoglicemia, hiperglicemia. hipertrigliceridemia); en 9 casos ocurrieron sepsis relacionada al catéter, en algunos hasta tres episodios y en 7 colestasia. El costo promedio de la alimentación parenteral para el grupo estudiado fue de $\$ 10162$ diarios (U\$ 25). Es necesario contar con equipos especializados y normas adecuadas para el manejo de los niños con intestjno corto.

(Palabras clave: intestino corto, alimentación parcnteral.)

\section{Referencias}

1. Collins IB. Georgesm KE, Vicenfe $Y$, Kelly $D R$, Figeterou $R$ : Short bowel syndrome. Seminirs Pediatr Suig 1995: 4: 60.73.

2. Georgeson KE, Biesux CW: Outcome and intestinal adaptation in neonatal short-bowel syndrome. J Pediatr Surg 1992: 27: 344-350.

3. Dorney SFA, Ament ME, Berquist WE, Yargas JH, Harseilf $E$ : Improved survival in very short bowel of infancy with use of long-ter'm parenteral nutrition. J Pedialr 1985: 107: 521-525.

4. Goulet $O J$, Revitlon $Y$, Jan $D$. et at: Neonatal short bowel syndrome. J Pediatr 1991; 119: 18-23.

5. Norlgatrd I. Stenhaek Hansen B, Brobech Mortensen $P$ : Colon as a digestive organ in patients with shor bowel. Lancet 1994: 343: 373-376

6. Weberhan $S$ : Carbohydrates vs fat in the dietary treatment of short bowel syndrome. Nulr Rev 1994: 52: 354-356

7. Chtel MS, Ftrrell MK, Ziegler MM. Warner BW: Intensive nutritional support and remedial surgical intervention for extreme shorl bowel syndrome. J Pediatr Gastroenterol Nutr 1994: 19: 295-298.

8. Muphy LAf, Limmeth TO: Central venous catheter care in parenteral nutrition: A review. JPEN 1987; 11: 190-201.

y. Gorrisom RN, Wilson MA: lntravenous and central calheter infections. Surg Clin North Am 1994; 74: $557-570$

10. Whatef CL. Hatom K. Feldmun JG, Rubin LG: Infection tates of Broviac-Hickman catheters and implantable venous devices. Am J Dis Child 1988: 142: 536-540.

11. Vargeis JH. ArteH ME: Berquist WE: Long-term home parenteral nutrition in Pediatrics: ten years of experience in 102 palients. J Pediatr Gastroenterol Nutr 1987:6: 24-32.

12. Chutut-Has L, Rossi TM, Heilinger LA. Lerner A, Riddletberger MM, Lebenthat E: Nutritional assessinent of children with short-bowel syndrome receiving borte parenteral nulrition. Am J Dis Cbild 1987; 14]; 1093-1098

13. Moss RL, Das JB, Ruffensperger JG: Total parenteral nutrition-associated cholestasis: Clinucal and hystopathologic correlation. J Pediarr Surg 1993; 28: 12701275

14. Hofmeden AF: Defective biliary secretion during total parenteral nutrition: probable mechanisms and possible solutions. J Pediate Gastroenterol Nutr 1995 ; 20: $376-390$.

15. Rintala RJ. Liduhl H. Pohiounomi $M$ : Total parenterat nutrition-associated cholestasis in surgical neonates may be reversed by cholecystokinin: a preliminary report. J Pediatr Surg 199.5; 30: 827.830. 